BMJ Open

Diabetes

Research

\& Care

\title{
Problem-solving therapy for adults with diabetic retinopathy and diabetes- specific distress: a pilot randomized controlled trial
}

\author{
Gwyneth Rees, ${ }^{1}$ Fleur O'Hare, ${ }^{1}$ Marian Saeed, ${ }^{1}$ Bronwyn Sudholz, ${ }^{2,3}$ \\ Bonnie A Sturrock, ${ }^{1}$ Jing Xie, ${ }^{1}$ Jane Speight, ${ }^{3,4,5}$ Ecosse L Lamoureux ${ }^{1,6,7}$
}

To cite: Rees G, O'Hare F, Saeed M, et al. Problemsolving therapy for adults with diabetic retinopathy and diabetes-specific distress: a pilot randomized controlled trial. BMJ Open Diabetes Research and Care 2017;5: e000307. doi:10.1136/ bmjdrc-2016-000307

Received 3 August 2016 Revised 22 December 2016 Accepted 26 December 2016

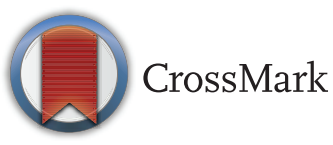

For numbered affiliations see end of article.

Correspondence to Dr Gwyneth Rees; grees@unimelb.edu.au

\section{ABSTRACT}

Objective: To provide preliminary evidence for the impact of problem-solving therapy for diabetes (PST-D) in adults with diabetic retinopathy (DR) and diabetes distress.

Research design and methods: In a pilot randomized controlled trial, 40 participants with DR and diabetes distress were allocated to the PST-D or control groups. Diabetes distress (DDS), depressive symptoms (PHQ-9), self-care activities (SDSCA), and $\mathrm{HbA}_{1 \mathrm{c}}$ were assessed at baseline, and 3 and 6-month follow-ups.

Results: At the 6-month follow-up, the PST-D group showed significant improvements relative to the control group, in 'regimen-related distress' (PST-D: $-1.3 \pm 1.4$; control: $-0.4 \pm 1.1$ ), depressive symptoms (PST-D: $-4.3 \pm 6.1$; control: $-0.3 \pm 4.6$ ), and $\mathrm{HbA}_{1 \mathrm{c}}$ (PST-D: $-1.2 \% \pm 1.01$; control: $0.2 \% \pm 1.2 \%$ ) (all $p<0.05$ ). In multiple regression analysis, adjusting for baseline values and sociodemographic factors, PST-D was associated with significant improvement in 'regimenrelated distress', depressive symptoms, and $\mathrm{HbA}_{1 \mathrm{c}}$ at the 6-month follow-up $(p<0.05)$.

Conclusions: PST-D is a promising intervention for improving psychological outcomes and glycemic control. A fully powered study is required to confirm these findings and examine mechanisms of change in HbA1c.

Trial registration number: ACTRN12616001010482; results.

\section{INTRODUCTION}

There is overwhelming evidence that diabetes distress and depressive symptoms are common in people with diabetes and have detrimental implications for quality of life, family functioning, diabetes outcomes, and healthcare costs. ${ }^{1}$ Rates of depression are higher in those with complications of diabetes $^{2}$ and prospective studies have identified that depression is a risk factor for the progression of diabetic microvascular complications. ${ }^{3}$ For example, depressive symptoms were related to a threefold increased risk of

\section{Significance of this study}

What is already known about this subject?

- We know that diabetes distress and depressive symptoms are common in people with diabetes and have a huge impact on many aspects of life.

- Evidence-based low-intensity psychological treatments exist but are not targeted or easily available within diabetes services.

What are the new findings

- This study provides preliminary evidence that problem-solving therapy tailored to diabetes can improve diabetes distress, depressive symptoms, and $\mathrm{HbA}_{1 \mathrm{c}}$ in people with diabetic retinopathy who are distressed by diabetes.

How might these results change the focus of research or clinical practice?

- These findings highlight an approach that could be delivered by staff in the diabetes or primary care team allowing full integration into existing services.

- Further research is needed to examine the causal mechanisms underlying change in $\mathrm{HbA}_{1 \mathrm{c}}$ and determine the longer term impact on psychological and clinical outcomes.

- Practical issues regarding implementation of problem-solving therapy tailored to diabetes as an integrated component of diabetes services require investigation.

progression from non-proliferative diabetic retinopathy (NPDR) to proliferative DR (PDR) in a 6-year period. ${ }^{3}$

Recent reviews and meta-analyses of depression interventions in diabetes have found few studies that have shown simultaneous improvement in psychological outcomes and $\mathrm{HbA}_{1 \mathrm{c} .}{ }^{4}$ This may be because depression treatments are often delivered in isolation from diabetes care. An integrated approach that focuses on depressive symptoms and diabetes distress in the context of diabetes management is likely to be more effective. Indeed, 
diabetes distress is more common than depression in people with diabetes, and more closely associated with diabetes self-management and glycemic control. ${ }^{56}$ This has led to the hypothesis that psychological interventions, which specifically address diabetes and are integrated in diabetes care, could improve psychological and biomedical outcomes in those with high levels of diabetes distress. ${ }^{5}{ }^{6}$ Evidence from a large trial of stepped care for depression in people with diabetes suggests that the greatest gains were obtained by those people presenting with micro and macrovascular complications. ${ }^{7}$ To date though, few studies have specifically focused on diabetes distress, ${ }^{8}$ and no study has targeted psychological interventions tailored for people with DR. The implications are clear: interventions that target the distress associated with diabetes as well as general depressive symptoms are likely to be most effective in improving mental health and clinical outcomes. However, this is yet to be tested empirically in those with the highest rates of depressive symptoms, that is, people with microvascular complications. To address this need, we recently adapted an evidence-based treatment for depression, namely 'problem-solving therapy for primary care' (PST-PC), ${ }^{9}$ to target diabetes-related problems that cause distress in people with DR. We chose to adapt PST-PC because it can be delivered by clinicians without specific mental health qualifications, enabling us to deliver the intervention via health professionals such as diabetes educators, eye care health professionals, or other allied health professionals responsible for the care of individuals with diabetes and DR. We tailored PST-PC to focus on problem-solving skill development to address specific barriers to diabetes management and aspects of diabetes and its complications that cause distress. Therefore, our approach enabled participants to identify and address challenges they experience in living with, and managing diabetes, as well as achieve the generic problem-solving skills and behavioral activation that reduces depression.

We hypothesized that this intervention would reduce diabetes distress and depressive symptoms; and improve diabetes self-care and $\mathrm{HbA}_{1 \mathrm{c}}$. We chose to focus on diabetes distress as our primary outcome (and inclusion criterion) rather than heightened depressive symptoms, since diabetes distress is more common and maybe more amenable to change through interventions that can be delivered in non-mental health setting. ${ }^{8}$ However, given that our intervention was an adaptation of an evidencebased intervention for depression, depressive symptoms were investigated as a secondary outcome measure. The aim of this pilot study was to establish preliminary evidence for the efficacy of diabetes-specific PST (PST-D).

\section{RESEARCH DESIGN AND METHODS}

Recruitment and eligibility

Forty participants were recruited from retinal clinics at the Royal Victoria Eye and Ear Hospital (RVEEH), Melbourne, Australia. A sample size of 40 was chosen for pragmatic reasons to be a feasible recruitment target for a pilot study. Inclusion criteria were (1) diagnosis of type 2 diabetes; (2) confirmed diagnosis of DR; (3) at least moderate diabetes-specific distress on the Diabetes Distress Scale (DDS) (overall score $\geq 2.0),{ }^{10}$ (4) at least 18 years old. Exclusion criteria were (1) insufficient English language skills to engage in PST-D sessions and the research interviews as determined by the research assistant; (2) insufficient cognitive ability to engage in PST-D as indicated by the 6-item Cognitive Impairment Test. $^{11}$

Medical files were screened and individuals who could be identified as having type 2 diabetes and DR were approached at the clinic or called by telephone to discuss the study. All potential participants were provided with a plain language statement and provided written consent to participate in the project. Ethics approval was obtained from the Royal Victorian Eye and Ear Hospital's Human Research Ethics Committee (HREC; project number 08/815H). Potential participants were invited to attend an appointment in which eligibility criteria were assessed and a retinal image of each eye obtained for grading to confirm DR diagnosis. Those individuals who met the study eligibility criteria completed a baseline assessment.

\section{Data collection}

Retinal photographs were obtained from two-field fundus photographs (Canon CR6-45NM, Canon, Japan) and clinical signs of DR graded following the modified Airlie House classification system. ${ }^{12}$

All assessments were conducted by a research assistant blinded to allocation. At baseline, clinical assessments included $\mathrm{HbA}_{1 \mathrm{c}}$ collected by venepuncture and analyzed via a pathology service and presenting visual acuity using a $3 \mathrm{~m}$ logarithm of the minimum angle of resolution chart (LogMAR). Diabetes duration, medications, and sociodemographic details were assessed by self-report. Structured interviews were conducted at baseline, and then again 3 and 6 months later (1) 17-item diabetesspecific distress $\left(\right.$ DDS $\left.^{13}\right)$ which provides a total score and four subscales: 'physician-related distress', 'regimenrelated distress', 'diabetes-related interpersonal distress', and 'emotional burden'; (2) depressive symptoms (9-item Patient Health Questionnaire $\left.(\mathrm{PHQ}-9)^{14}\right)$; and (3) diabetes self-care behaviors (revised version of the Summary of Diabetes Self-Care Activities (SDSCA) ${ }^{15}$ ) which provides an overview of specific diabetes selfmanagement activities in the last 7 days (eg, general diet, specific diet, exercise, smoking, and blood glucose testing). $\mathrm{HbA}_{1 \mathrm{c}}$ was also assessed at all visits.

\section{Randomization and intervention groups}

After the baseline interview, all participants in both groups were provided with publically available information brochures on diabetes-specific topics produced by Diabetes Victoria (ie, Living well with type 2 diabetes, Managing stress and diabetes, Diabetes and the eyes, 
and Monitoring blood glucose levels). Following baseline assessment, participants were randomized to the PST-D intervention $(n=21)$ or control $(n=19)$ group using a computer-generated random number sequence, generated and concealed using sealed envelopes by a clinical trials expert (external to the study team). The control group received care as usual.

We adapted the PST manual so that participants focused on problems specifically related to their diabetes and DR. The PST-D intervention consisted of up to eight sessions, each lasting 45-60 min, and delivered via telephone or in person (depending on participant's availability and preference) by a research assistant trained in PST-D delivery, under the supervision of a clinical psychologist (BS). During the first session, the participant was introduced to PST-D, and the rationale explained in the context of diabetes, complications, and distress. The interventionist then worked with the participant to identify problems related specifically to diabetes and its complications. This was facilitated initially by discussing participants' responses on the DDS and subsequently the development of a problem list related to diabetes across all domains of life. In all sessions, the participant chose a specific diabetes-related problem to work on and was guided through the problem-solving steps: (a) clarifying and defining the problem; (b) setting a realistic goal; (c) brainstorming multiple solution alternatives; (d) evaluating each solution for its advantages and obstacles to implementation; (e) choosing a preferred solution; (f) devising a specific action plan to implement the solution; and ( $\mathrm{g}$ ) evaluating outcomes from the previous session (sessions 2-8). Also, each session included activity scheduling, whereby participants make specific plans to engage in enjoyable activities, which are concordant with optimizing diabetes management during the following week. Sessions all followed the same structure with the interventionist working toward ensuring that the participant was taking a more active role in leading the problem-solving process as sessions progressed.

\section{Statistical analyses}

Univariate analysis assessed for group differences between participant characteristics at baseline and changes in outcome measures at 3 and 6 months. Controlling for confounders (baseline values, age, gender, duration of diabetes, diabetes medication, visual acuity), multiple regression analyses were used to assess the impact of PST-D on outcome variables at 3 and 6-month follow-up. Post hoc power estimations were conducted to determine the power to detect an intervention effect, and based on our 6-month follow-up with PST intervention and six covariates noted above in the multiple linear regression analysis. We estimated the power based on the changes in the adjusted $R^{2}$ when the intervention is included in the model. For PHQ-9, the $R^{2}$ is $48.01 \%$ for the full model (including the intervention group) and $39.00 \%$ for the reduced model (excluding the intervention group). The $\mathrm{R}^{2}$ change is 0.09 , with a sample size of 34 and a significance level of 0.05 , we had 45.30 power to detect the difference between the PST intervention and usual care group. The power to detect an intervention effect on HbA1c and DDS was $92 \%$ and $12 \%$, respectively.

Univariate analysis assessed for group differences at baseline between participants in the intervention group who completed follow-up assessments and those who were lost to follow-up.

\section{RESULTS}

The mean age of participants was $59.9 \pm 7.8$ years and $67.5 \%(n=27)$ were men. The average duration of type 2 diabetes was $21.0 \pm 16.0$ years, and $\mathrm{HbA}_{1 \mathrm{c}}$ was $8.2 \pm 1.6 \%$ $(65.6 \mathrm{mmol} / \mathrm{mol})$. At baseline, the PST-D and control groups were comparable in terms of sociodemographic and clinical characteristics, as well depressive symptoms and diabetes distress scores, except for the DDS 'diabetes-related interpersonal distress' subscale which was significantly higher in the PST-D group than the control group (PST-D: $3.0 \pm 1.2$; control: $2.5 \pm 1.2, \mathrm{p}=0.009$ ) (table 1).

Two participants declined further PST-D after one session. One participant received only four PST-D sessions due to illness. The remaining 18 participants received between 5 and 8 sessions. Six participants in the PST-D group did not attend the final follow-up, with an additional two declining the final $\mathrm{HbA}_{1 \mathrm{c}}$ assessment. All control group participants attended the follow-up assessments; although at each follow-up, $\mathrm{HbA}_{1 \mathrm{c}}$ was not obtained from one participant. Compared with participants in the intervention group who completed the study, those who were lost to follow-up assessments had lower scores on the DDS 'regimen-related distress' subscale at baseline (PST-D completers: $3.4 \pm 1.4$; PST-D lost to follow-up: $2.1 \pm 0.9 ; \mathrm{p}=0.04)$. There was also a trend to suggest that those lost to follow-up were more likely to be living alone $(60 \%)$ than those who completed all follow-up assessments (13.3\%; $\mathrm{p}=0.07)$. No other differences were observed.

At 3 months, there was a non-significant trend, suggesting a slightly greater reduction in 'regimen-related distress' in the PST-D group $(-1.2 \pm 1.2)$ relative to the control group $(-0.4 \pm 1.4 ; \mathrm{p}=.08)$. At the 6-month follow-up, the between-group difference in change scores reached statistical significance (PST-D: $-1.3 \pm 1.4$; control: $-0.4 \pm 1.1 ; \mathrm{p}=0.03)$. Compared with the control group, the PST-D group also showed a significantly greater reduction at 3 months in 'diabetes-related interpersonal distress' relative to the control group which demonstrated an increase (PST-D: $-0.9 \pm 1.4$; control: 0.2 $\pm 1.1 ; \mathrm{p}=0.01)$. Depressive symptoms reduced significantly more so in the intervention compared with control group at 3-month follow-up (PST-D: $-3.8 \pm 3.0$; control: $-1.1 \pm 3.9 ; \mathrm{p}=0.03)$. This latter difference was higher at 6 months with the PST-D group showing a $-4.3 \pm 6.1$ 
Table 1 Participants' characteristics at baseline and 3 and 6-month primary and secondary outcomes

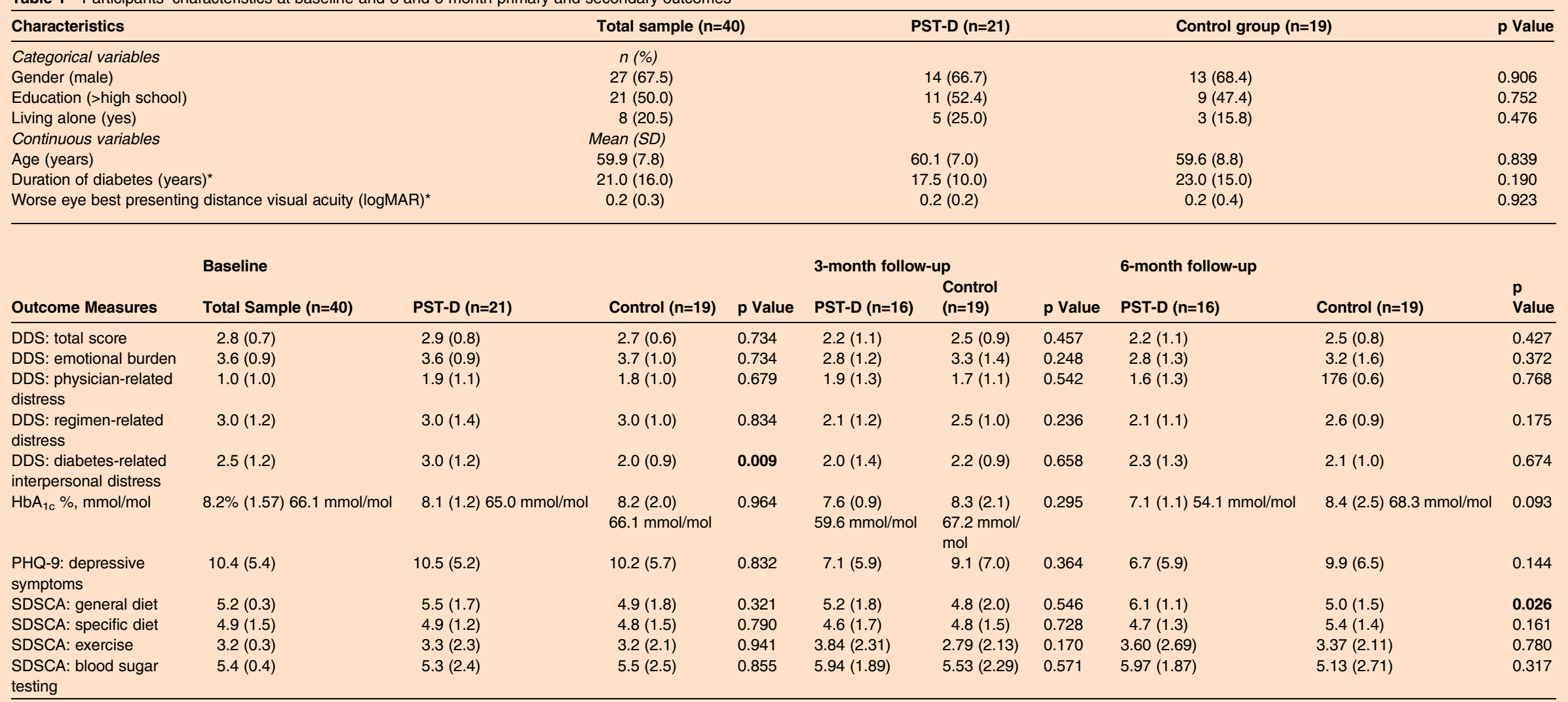

Bold indicates significance.

*Median (IQR).

DDS, Diabetes Distress Scale; $\mathrm{HbA}_{1 \mathrm{c}}$, hemoglobin $\mathrm{A}_{1 \mathrm{c}}$ (average blood glucose over 12 weeks); logMAR, logarithm of the minimum angle of resolution; PHQ-9, Patient Health Questionnaire 9; SDSCA, Summary of Diabetes Self-Care Activities. 
reduction on the PHQ-9 from baseline relative to -0.3 \pm 4.6 in the control group $(\mathrm{p}=0.04)$. For diabetes selfcare, no significant between-group differences in change scores were found at 3 or 6 months, although a trend for increased frequency in self-monitoring of blood glucose in the PST-D group relative to the control group at 6 months was identified (PST_D: 1.2 \pm 2.7 ; control: $-0.3 \pm 2.5 ; \mathrm{p}=0.09$ ). At 6 months, there was a significant between-group difference in change in $\mathrm{HbA}_{1 \mathrm{c}}$ from baseline representing a reduction in the intervention group $(-1.2 \% \pm 1.01)$ with no change in the control group $(0.2 \% \pm 1.2 \%) \quad(p=0.002)$.

Multivariate analysis, adjusting for baseline values, sociodemographic, and clinical factors (age, gender, duration of diabetes, diabetes medication, visual acuity at baseline), identified a significant impact of PST-D on 'regimen-related distress', $\mathrm{HbA}_{1 \mathrm{c}}$, and depressive symptoms at the 6 -month visit $(\mathrm{p}<0.05$; table 2$)$.

\section{CONCLUSIONS}

This is the first pilot randomized controlled trial to examine the effectiveness of a diabetes-specific problem-solving intervention in adults with DR, experiencing at least moderate diabetes distress. Our findings provide preliminary evidence that this intervention may improve diabetes distress, particularly associated with the diabetes regimen. Furthermore, our results suggest that PST-D has the potential to improve depressive symptoms and produce a clinically significant reduction in $\mathrm{HbAl}_{\mathrm{c}}$ of $-1.2 \%$. These are important data as few interventions for people with diabetes have been able to improve psychological and clinical outcomes. ${ }^{16}$ Targeting this intervention to individuals with early-stage $\mathrm{DR}$ and distress may also offer longer term outcomes, given that improved glycemic control in this group may delay the progression of NPDR to the vision-threatening stage. ${ }^{17} \mathrm{~A}$ benefit of the diabetes-specific PST is that it is designed to be delivered by diabetes or primary care clinicians, rather than by mental health specialists. Therefore, with brief training, existing members of the diabetes or primary care team could deliver this intervention. In addition, PST-D could be delivered in accessible formats, including telephone or online.

Consistent with emerging evidence, our study suggests that addressing the distress associated with diabetes management may impact on wider outcomes, such as depressive symptoms and glycemic control. For example, the REDEEM trial showed that reductions in 'regimenrelated distress' were associated with improved diabetes self-management and glycemic control. ${ }^{18}$ However, we found no significant effect on self-management behaviors, that is, diet and exercise as assessed by the SDSCA. One explanation is that the SDSCA may not have been sufficiently responsive to the PST-D intervention. Notably, we did not assess diabetes medication taking behaviors, which may have impacted on glycemic control. Hence, the mechanisms underlying change in 
$\mathrm{HbA}_{1 \mathrm{c}}$, which may be due to a range of neuroendocrine, inflammatory, or behavioral pathways, require further exploration in a fully powered study. In addition, only the 'regimen-related distress' subscale of the DDS showed a significant change. This may be due to the study's lack of power. Post hoc power estimations revealed this study has sufficient power to detect differences as a result of the intervention on HbAlc, but lower power for the PHQ-9 and DDS. Our findings may also reflect poor precision of the DDS total score and other subscales, as indicated by our recent Rasch analysis of the DDS. ${ }^{19}$ A fully powered study with a psychometrically sound assessment of diabetes distress is required to confirm these findings.

Our pilot study was limited by a small sample size and was potentially underpowered. All participants were retained in the control group, and engagement in PST-D was high; however, attrition in the intervention group attendance at follow-up assessments was lower (76\% and $71 \%$ attending for assessments at 3 and 6 months). While this is concerning, attrition in the range of $20-30 \%$ is considered acceptable in behavioral studies of this nature. ${ }^{20}$ Loss to follow-up may have been due to the burden of participation, or it may represent a self-selection bias skewing results. Indeed, we found that those who dropped out had lower levels of regimen-related distress at baseline. This may suggest that those with lower regimen-related distress may need less intensive intervention. That those who dropped out showed a trend to be living alone may indicate the importance of social support in the uptake of such interventions and may point to the need for alternative delivery options (eg, group-based programs). This requires further examination in future studies. A qualitative study would worthwhile to explore reasons for attrition as well as the applicability and utility of the PST-D in participants' daily lives. Our sample also included a higher proportion of men than women, which is unusual and may indicate recruitment bias, given that previous research has indicated higher levels of diabetes distress in women. ${ }^{21}$ Research has also indicated that gender differences in the association between diabetes distress and health behaviors (eg, physical activity, smoking ${ }^{21}$ which requires investigation in a full-scaled study. Finally, our study included a 'usual care' control group rather than an active (support/attention) condition controlling for nonspecific intervention effects.

In conclusion, these preliminary findings suggest the potential of PST-D, but a fully powered study is needed to: (1) confirm these findings, (2) test the causal mechanisms underlying change in $\mathrm{HbA}_{1 \mathrm{c}}$ taking into account gender differences; (3) determine longer term impact on psychological, diabetes, and DR outcomes; (4) examine the cost-effectiveness of this approach; and (5) explore the practical issues regarding implementation as an integrated component of diabetes services.
Author affiliations

${ }^{1}$ Centre for Eye Research Australia, Royal Victorian Eye and Ear Hospital, University of Melbourne, Melbourne, Australia

${ }^{2}$ Deakin University, Institute for Physical Activity and Nutrition (IPAN), School of Exercise and Nutrition Sciences, Geelong, Australia

${ }^{3}$ Deakin University, School of Psychology, Geelong, Australia

${ }^{4}$ The Australian Centre for Behavioural Research in Diabetes, Diabetes Victoria Melbourne, Victoria, Australia

${ }^{5}$ AHP Research, Hornchurch, UK

${ }^{6}$ Singapore Eye Research Institute, National University of Singapore,

Singapore, Singapore

${ }^{7}$ Duke-NUS Medical School, Singapore, Singapore

Acknowledgements This research was supported by an Australia Research Council Linkage grant (LP0884108, Principle Investigator ELL) with support from industry partners the Royal Victorian Eye and Ear Hospital and Diabetes Australia-Victoria (now Diabetes Victoria). GR holds a NHMRC Career Development Fellowship 2014-2017. CERA receives Operational Infrastructure Support from the Victorian Government. JS is supported by core funding to the ACBRD from Diabetes Victoria and Deakin University.

Contributors GR and ELL conceived the study. FO, GR, and JS wrote the protocol. GR, JS, and BAS adapted the PST-PC manual for this population (PST-D). GR and BAS trained and supervised the staff in the implementation of PST-D. FO, MS, and BAS collected the data. JX conducted statistical analysis. GR drafted the manuscript. All authors reviewed/edited the manuscript and approve the final version. JX is the guarantor of this work and, as such, had full access to all the study data and takes responsibility for the integrity of the data and the accuracy of the data analysis.

Funding Australian Research Council (grant number LP0884108).

Competing interests None declared.

Ethics approval Royal Victorian Eye and Ear Hospital HREC.

Provenance and peer review Not commissioned; externally peer reviewed.

Data sharing statement No additional data are available.

Open Access This is an Open Access article distributed in accordance with the Creative Commons Attribution Non Commercial (CC BY-NC 4.0) license, which permits others to distribute, remix, adapt, build upon this work noncommercially, and license their derivative works on different terms, provided the original work is properly cited and the use is non-commercial. See: http:// creativecommons.org/licenses/by-nc/4.0/

\section{REFERENCES}

1. Nicolucci A, Kovacs Burns K, Holt RI, et al. Diabetes Attitudes, Wishes and Needs second study (DAWN2 ${ }^{\mathrm{TM}}$ ): cross-national benchmarking of diabetes-related psychosocial outcomes for people with diabetes. Diabet Med 2013;30:767-77.

2. De Groot M, Anderson R, Freedland KE, et al. Association of depression and diabetes complications: a meta-analysis. Psychosom Med 2001;63:619-30.

3. Roy MS, Roy A, Affouf M. Depression is a risk factor for poor glycemic control and retinopathy in African-Americans with type 1 diabetes. Psychosom Med 2007;69:537-42.

4. Harkness E, Macdonald W, Valderas J, et al. Identifying psychosocial interventions that improve both physical and mental health in patients with diabetes a systematic review and meta-analysis. Diabetes Care 2010;33:926-30.

5. Fisher L, Mullan JT, Arean P, et al. Diabetes distress but not clinical depression or depressive symptoms is associated with glycemic control in both cross-sectional and longitudinal analyses. Diabetes Care 2010;33:23-8.

6. Van Bastelaar KM, Pouwer F, Geelhoed-Duijvestijn PH, et al. Diabetes-specific emotional distress mediates the association between depressive symptoms and glycaemic control in Type 1 and Type 2 diabetes. Diabet Med 2010;27:798-803.

7. Kinder LS, Katon WJ, Ludman E, et al. Improving depression care in patients with diabetes and multiple complications. J Gen Intern Med 2006;21:1036-41.

8. Sturt J, Dennick K, Hessler D, et al. Effective interventions for reducing diabetes distress: systematic review and meta-analysis. Int Diabetes Nurs 2015;12:40-55. 
9. Hegel M, Barrett J, Oxman T, et al. Problem-solving treatment for primary care (PST-PC): a treatment manual for depression. Hanover, $\mathrm{NH}$ : Dartmouth University, 1999.

10. Fisher L, Hessler DM, Polonsky WH, et al. When is diabetes distress clinically meaningful? Establishing cut points for the Diabetes Distress Scale. Diabetes Care 2012;35:259-64.

11. Brooke P, Bullock R. Validation of a 6 Item Cognitive Impairment Test with a view to primary care usage. Int $J$ Geriatr Psychiatry 1999;14:936-40.

12. Early Treatment Diabetic Retinopathy Study Research Group. Grading diabetic-retinopathy from stereoscopic color fundus photographs-an extension of the modified Airlie House classification-ETDRS report number-10. Ophthalmology 1991;98(5 Suppl):786-806.

13. Fisher L, Glasgow RE, Mullan JT, et al. Development of a brief diabetes distress screening instrument. Ann Fam Med 2008;6:246-52.

14. Kroenke K, Spitzer RL, Williams JB. The Phq-9. J Gen Intern Med 2001:16:606-13.

15. Toobert DJ, Hampson SE, Glasgow RE. The summary of diabetes self-care activities measure: results from 7 studies and a revised scale. Diabetes Care 2000;23:943-50.
16. Markowitz SM, Gonzalez JS, Wilkinson JL, et al. A review of treating depression in diabetes: emerging findings. Psychosomatics 2011;52:1-18.

17. Chew EY, Ambrosius WT, Davis MD, et al., The ACCORD Study Group and ACCORD Eye Study Group. Effects of medical therapies on retinopathy progression in type 2 diabetes. $N$ Engl J Med 2010;363:233-44.

18. Hessler D, Fisher L, Glasgow RE, et al. Reductions in regimen distress are associated with improved management and glycemic control over time. Diabetes Care 2014;37:617-24.

19. Fenwick EK, Rees G, Holmes-Truscott E, et al. What is the best measure for assessing diabetes distress? A comparison of the Problem Areas in Diabetes and Diabetes Distress Scale: results from Diabetes MILES-Australia. J Health Psychol 2016.

20. Fisher L, Hessler D, Naranjo D, et al. AASAP: a program to increase recruitment and retention in clinical trials. Patient Educ Couns 2012;86:372-7.

21. Lipscombe C, Smith KJ, Gariepy G, et al. Gender differences in the association between lifestyle behaviors and diabetes distress in a community sample of adults with type 2 diabetes. J Diabetes 2016;8:269-78. 